\title{
New Julia Sets of Ishikawa Iterates
}

\author{
Yashwant S Chauhan \\ Asst. Professor \\ Computer Science \& Engg. \\ Department \\ G. B. Pant Engg. College, \\ Pauri Garhwal
}

\author{
Rajeshri Rana \\ Asst. Professor \\ Applied Science and Humanities \\ Department \\ G. B. Pant Engg. College, \\ Pauri Garhwal
}

\author{
Ashish Negi \\ Asst. Professor \\ Computer Science \& Engg. \\ Department \\ G. B. Pant Engg. College, \\ Pauri Garhwal
}

\begin{abstract}
We investigate in this paper the dynamics and the method of generating fractal images for Ishikawa iteration procedure. The geometry of relative superior Julia sets are explored for Ishikawa iteration.
\end{abstract}

\section{Keywords}

Complex dynamics, relative superior Julia Set, Ishikawa Iteration.

\section{INTRODUCTION}

Many authors have presented the papers on several "orbit traps" rendering methods to create the artistic fractal images. An orbit trap is a bounded area in complex plane into which an orbiting point may fall. Motivated by this idea of "orbit traps", this paper introduces the different types of orbit traps for Ishikawa iteration procedure. It is well known that Julia sets of $z_{n+1}=a z_{n}^{2}+c$ are connected and bounded for $a$ and $c$. So, we consider them as orbit traps and explore their relative fractal images.

\section{PRELIMINARIES}

Let $\left\{z_{n}: n=1,2,3,4 \ldots \ldots \ldots\right\}$, denoted by $\left\{z_{n}\right\}$ be a

sequence of complex numbers. Then, we say $\operatorname{Lim}_{n \rightarrow \infty} z_{n}=\infty$ if for given $\mathrm{M}>0$, there exists $\mathrm{N}>0$, such that for all $\mathrm{n}>\mathrm{N}$, we must have $\left|z_{n}\right|>M$. Thus all the values of $z_{n}$, lies outside a circle of radius $M$, for sufficiently large values of $n$.

Let $Q(z)=a_{0} z^{n}+a_{1} z^{n-1}+a_{2} z^{n-2}+\ldots \ldots \ldots \ldots \ldots+a_{n-1} z^{1}+a_{n} z^{0} ; a_{0} \neq 0$ be a polynomial of degree $\mathrm{n}$, where $n \geq 2$. The coefficients are allowed to be complex numbers. In other words, it follows that $Q_{c}(z)=z^{2}+c$.

Definition 2.1: Let $X$ be a nonempty set and $f: X \rightarrow X$. For any point $x_{0} \in X$, the Picard's orbit is defined as the set of

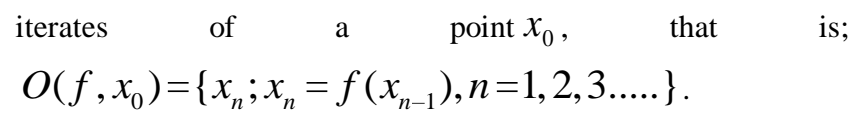

In functional dynamics, we have existence of two different types of points. Points that leave the interval after a finite number are in stable set of infinity. Points that never leave the interval after any number of iterations have bounded orbits.
So, an orbit is bounded if there exists a positive real number, such that the modulus of every point in the orbit is less than this number. The collection of points that are bounded, i.e. there exists $\mathrm{M}$, such that $\left|Q^{n}(z)\right| \leq M$, for all $\mathrm{n}$, is called as a prisoner set while the collection of points that are in the stable set of infinity is called the escape set. Hence, the boundary of the prisoner set is simultaneously the boundary of escape set and that is Julia set for Q.

Definition 2.2: The set of points $\mathrm{K}$ whose orbits are bounded under the iteration function of $Q_{c}(z)$ is called the Julia set. We choose the initial point 0 , as 0 is the only critical point of $Q_{c}(z)$.

\section{ISHIKAWA ITERATION FOR RELATIVE SUPERIOR JULIA SETS}

Let $X$ be a subset of real or complex numbers and $f: X \rightarrow X$. For $x_{0} \in X$, we construct the sequences $\left\{x_{n}\right\}$ and $\left\{y_{n}\right\}$ in $\mathrm{X}$ in the following manner:

$$
\begin{aligned}
& y_{0}=s_{0}^{\prime} f\left(x_{0}\right)+\left(1-s_{0}^{\prime}\right) x_{0} \\
& y_{1}=s_{1}^{\prime} f\left(x_{1}\right)+\left(1-s_{1}^{\prime}\right) x_{1} \ldots \ldots \\
& y_{n}=s_{n}^{\prime} f\left(x_{n}\right)+\left(1-s_{n}^{\prime}\right) x_{n}
\end{aligned}
$$

where $0 \leq s_{n}^{\prime} \leq 1$ and $s_{n}^{\prime}$ is convergent to non zero number and

$x_{1}=s_{0} f\left(y_{0}\right)+\left(1-s_{0}\right) x_{0}$

$x_{2}=s_{1} f\left(y_{1}\right)+\left(1-s_{1}\right) x_{1} \ldots \ldots$

$x_{n}=s_{n-1} f\left(y_{n-1}\right)+\left(1-s_{n-1}\right) x_{n-1}$

where $0 \leq s_{n} \leq 1$ and $s_{n}$ is convergent to non zero number[12].

Definition 3.1: The sequences $x_{n}$ and $y_{n}$ constructed above is called Ishikawa sequences of iteration or relative superior sequences of iterates. We denote it by $R S O\left(x_{0}, s_{n}, s_{n}^{\prime}, t\right)$.

Notice that $\operatorname{RSO}\left(x_{0}, s_{n}, s_{n}^{\prime}, t\right)$ with $s_{n}^{\prime}=1$ is $\operatorname{RSO}\left(x_{0}, s_{n}, t\right)$ 
i.e. Mann's orbit and if we place $s_{n}=s_{n}^{\prime}=1$ then $R S O\left(x_{0}, s_{n}, s_{n}^{\prime}, t\right)$ reduces to $O\left(x_{0}, t\right)$.We remark that Ishikawa orbit $\operatorname{RSO}\left(x_{0}, s_{n}, s_{n}^{\prime}, t\right)$ with $s_{n}^{\prime}=1 / 2$ is Relative superior orbit. Now we define Julia set for function with respect to Ishikawa iterates. We call them as Relative Superior Julia sets.

Definition 3.2: The set of points SK whose orbits are bounded under Relative superior iteration of function $\mathrm{Q}(\mathrm{z})$ is called Relative Superior Julia sets. Relative Superior Julia set of Q is boundary of Julia set RSK.

We now define escape criterions for these sets.

\subsection{Relative Superior Escape Criterions for Quadratics}

The following theorem gives us an escape Criterions for function $Q_{c}=z^{2}+c$ in respect to Ishikawa iteration procedure.

Theorem 3.1: Let's assume that $|z| \geq|c|>2 / s$; $|z| \geq|c|>2 / s^{\prime}$, where $0<s<1, \quad 0<s^{\prime}<1$ and $c$ is a complex number. Define $z_{1}=(1-s) z+s Q_{c}(z)$

$$
\begin{aligned}
& \text { : } \\
& z_{n}=(1-s) z_{n-1}+s Q_{c}\left(z_{n-1}\right)
\end{aligned}
$$

where $Q_{c}(z)$ can be a quadratic, cubic or biquadratic polynomial in terms of $s^{\prime}$ and $\mathrm{n}=2,3,4, \ldots$ then $\left|z_{n}\right| \rightarrow \infty$, as $n \rightarrow \infty$.

Proof: Let's take $\left|Q_{c}(z)\right|=\left|\left(1-s^{\prime}\right) z+s^{\prime} Q_{c}^{\prime}(z)\right|$, where $Q_{c}^{\prime}(z)=z^{2}+c$

$$
\begin{aligned}
& =\left|s^{\prime} z^{2}+\left(1-s^{\prime}\right) z+s^{\prime} c\right| \\
& \geq\left|s^{\prime} z^{2}+\left(1-s^{\prime}\right) z\right|-\left|s^{\prime} c\right| \\
& \geq|z|\left(\left|s^{\prime} z+\left(1-s^{\prime}\right)\right|\right)-s^{\prime}|z| \\
& \geq|z|\left(\left|s^{\prime} z\right|-1+s^{\prime}\right)-s^{\prime}|z| \\
& =|z|\left(\left|s^{\prime} z\right|-1\right)
\end{aligned}
$$$$
\geq|z|\left(\left|s^{\prime} z+\left(1-s^{\prime}\right)\right|\right)-s^{\prime}|z| \quad(\because|z| \geq|c|)
$$

Now since, $\quad z_{n}=(1-s) z_{n-1}+s Q_{c}^{\prime}(z)$

$$
\text { So, } \begin{aligned}
\left|z_{1}\right| & =\left|(1-s) z+s Q_{c}(z)\right| \quad \text { on substituting (1) } \\
& =|(1-s) z+s| z\left|\left(\left|s^{\prime} z\right|-1\right)\right| \\
& =|z-s z+s| z|\cdot| s^{\prime} z|-s| z|| \\
& \geq(|z|+|s z|)+\left(s|z| \cdot\left|s^{\prime} z\right|-s|z|\right) \\
& \geq|z|+|s z|+s|z| \cdot\left|s^{\prime} z\right|-s|z| \\
\geq|z|(1+ & \left.s s^{\prime}|z|\right), \text { since } s|z|>2, \text { so, } s s^{\prime}|z|>2 \text {, there }
\end{aligned}
$$
exists $\lambda>0$, such that $s s^{\prime}|z|-1>1+\lambda$

$$
\text { Consequently } \quad\left|z_{1}\right|>(1+\lambda)|z|
$$

$$
\begin{aligned}
& \vdots \\
& z_{n}\left|>(1+\lambda)^{n}\right| z \mid
\end{aligned}
$$

Thus, the Ishikawa orbit of $z$, under the quadratic function tends to infinity. This completes the proof.

Corollary 3.1: Suppose that $|c|>2 / s ;|c|>2 / s^{\prime}$.Then, the relative superior orbit of Ishikawa $\operatorname{RSO}\left(Q_{c}, 0, s, s^{\prime}\right)$ escapes to infinity.

In the proof of the theorem, we used the facts that $|z| \geq|c|$ and $|z|>2 / s$ as well as $|z|>2 / s^{\prime}$. Hence, the following corollary is the refinement of the escape criterion discussed in the above theorem.

Corollary 3.2(Escape Criterion): Suppose that $|z|>\max \left\{|c|, 2 / s, 2 / s^{\prime}\right\}$, then $\left|z_{n}\right|>(1+\lambda)^{n}|z|$ and $\left|z_{n}\right| \rightarrow \infty$ as $n \rightarrow \infty$.

Corollary 3.3: Suppose that $\left|z_{k}\right|>\max \left\{|c|, 2 / s, 2 / s^{\prime}\right\}$, for some $k \geq 0$.Then, $\quad\left|z_{k+1}\right|>(1+\lambda)^{n}\left|z_{k}\right| \quad$ and $\left|z_{n}\right| \rightarrow \infty$ as $n \rightarrow \infty$.

This corollary gives us an algorithm for computing the Relative Superior Julia sets of $Q_{c}$, for any c. Given any point $|z| \leq|c|$, we have computed the superior orbit of $z$. If for some $n$, $\left|z_{n}\right|$ lies outside the circle of radius $\max \left\{|c|, 2 / s, 2 / s^{\prime}\right\}$, we guarantee that the orbit escapes. Hence, $\mathrm{z}$ is not in the Relative Superior Julia sets. On the other hand, if $\left|z_{n}\right|$ never exceeds this bound, then by definition of the Relative Superior Julia sets, denoted by $R S K_{c}$. We can make extensive use of this algorithm in the next section.

3.2 Relative Superior Escape Criterion for Cubic Polynomials:

First, we prove the following theorem for the function $Q_{a, b}(z)=z^{3}+a z+b$ with respect to the Ishikawa iteration procedure.

Theorem $\quad 3.2: \quad$ Suppose $|z|>|b|>(|a|+2 / s)^{1 / 2}$, $|z|>|b|>\left(|a|+2 / s^{\prime}\right)^{1 / 2}$ exists, where $0<s \leq 1 ; 0<s^{\prime}<1$ and $a$ and $b$ are in complex plane .Define $z_{1}=(1-s) z+s Q_{a, b}(z)$

$$
z_{n}=(1-s) z_{n-1}+s Q_{a, b}\left(z_{n-1}\right), \mathrm{n}=2,3 \ldots \ldots
$$

where $Q_{a, b}(z)$ is the function of $s^{\prime}$, then $\left|z_{n}\right| \rightarrow \infty$ as $n \rightarrow \infty$.

Proof: Let's take $\left|Q_{a, b}(z)\right|=\left|\left(1-s^{\prime}\right) z+s^{\prime} Q_{a, b}^{\prime}(z)\right|$

$$
\begin{aligned}
& =\left|\left(1-s^{\prime}\right) z+s^{\prime}\left(z^{3}+a z+b\right)\right| \\
& =\left|s^{\prime} z^{3}+s^{\prime} a z+z-s^{\prime} z+b s^{\prime}\right|
\end{aligned}
$$




$$
\begin{aligned}
& \geq\left|s^{\prime} z^{3}+s^{\prime} a z+z-s^{\prime} z\right|-\left|b s^{\prime}\right| \\
& \geq|z|\left(\left|s^{\prime} z^{2}+s^{\prime} a+1-s^{\prime}\right|\right)-s^{\prime}|z| \quad \because|z| \geq|b| \\
& \geq|z|\left(\left|s^{\prime} z^{2}+a s^{\prime}\right|-\left|1-s^{\prime}\right|\right)-s^{\prime}|z| \\
& =|z|\left\{\left|s^{\prime} z^{2}+a s^{\prime}\right|-1+s^{\prime}-s^{\prime}\right\} \\
& =|z|\left\{s^{\prime}\left|z^{2}+a\right|-1\right\} \\
& =s^{\prime}|z|\left\{\left|z^{2}+a\right|-1 / s^{\prime}\right\} \\
& \geq s^{\prime}|z|\left\{|z|^{2}-|a|-1 / s^{\prime}\right\} \\
& =s^{\prime}|z|\left\{|z|^{2}-\left(|a|+1 / s^{\prime}\right)\right\}
\end{aligned}
$$$$
\text { Now since }\left|z_{1}\right|=\left|(1-s) z+s Q_{a, b}(z)\right|
$$$$
=|(1-s) z+s \cdot| z\left|\cdot\left\{s^{\prime}\left(\left|z^{2}+a\right|\right)-1\right\}\right|
$$$$
=|z-s z+s| z\left|\cdot s^{\prime}\left(\left|z^{2}+a\right|\right)-s\right| z||
$$$$
\geq|z|+s|z|+\left\{s|z| \cdot s^{\prime}\left(\left|z^{2}+a\right|\right)-s|z|\right\}
$$$$
\geq|z|+s|z|+s . s^{\prime}|z|\left(\left|z^{2}+a\right|\right)-s|z|
$$$$
\geq|z|\left\{1+s . s^{\prime}\left|z^{2}+a\right|\right\}
$$$$
=|z| s . s^{\prime}\left(1 / s . s^{\prime}+\left|z^{2}+a\right|\right)
$$$$
\geq|z| s . s^{\prime}\left(1 / s . s^{\prime}+\left|z^{2}\right|-|a|\right)
$$$$
=|z| s . s^{\prime}\left\{\left|z^{2}\right|-\left(|a|-1 / s . s^{\prime}\right)\right\}
$$

Since $|z|>(|a|+2 / s)^{1 / 2}$ and $|z|>\left(|a|+2 / s^{\prime}\right)^{1 / 2}$ exists and so $\quad|z|>\left(|a|+2 / s s^{\prime}\right)^{1 / 2}$ follows .Therefore, $\left|z^{2}\right|-\left(|a|+1 / s s^{\prime}\right)^{1 / 2}>1 / s s^{\prime}$ such

that $s s^{\prime}\left\{\left|z^{2}\right|-\left(|a|+1 / s s^{\prime}\right)\right\}>1$. Hence, there exists $\gamma>1$, such that $\left|z_{1}\right|>\gamma|z|$. Repeating this argument $\mathrm{n}$ time, we get $\left|z_{n}\right|>\gamma^{n}|z|$. Therefore, the Relative Superior orbit of $z$, under the cubic polynomial $Q_{a, b}(z)$, tends to infinity. This completes the proof.

Corollary 3.4: Suppose that $|b|>(|a|+2 / s)^{1 / 2}$ and $|b|>\left(|a|+2 / s^{\prime}\right)^{1 / 2}$ exists. Then, the Relative Superior orbit $R S O\left(Q_{a, b}, 0, s, s^{\prime}\right)$ escapes to infinity.

\section{Corollary 3.5(Escape Criterion):}

Suppose $\quad|z|>\max \left\{|b|,(|a|+2 / s)^{1 / 2},\left(|a|+2 / s^{\prime}\right)^{1 / 2}\right\}$ then $\left|z_{n}\right| \rightarrow \infty$ as $n \rightarrow \infty$. Corollary 3.5 gives an escape criterion for cubic polynomials.

\section{Corollary 3.6:}

Assume that $\left|z_{k}\right|>\max \left\{|b|,(|a|+2 / s)^{1 / 2},\left(|a|+2 / s^{\prime}\right)^{1 / 2}\right\}$ for some $k \geq 0$.Then $\left|z_{k+1}\right|>\gamma\left|z_{k}\right|$ and $\left|z_{n}\right| \rightarrow \infty$, as $n \rightarrow \infty$.From Corollary 3.6, we find an algorithm for computing the superior Julia sets of $Q_{a, b}(z)$, for any $a$ and $b$.

\subsection{A General Escape Criterion:}

We will obtain a general escape criterion for polynomials of the form $G_{c}(z)=z^{n}+c$.

Theorem 3.3: For general function $G_{c}(z)=z^{n}+c, \mathrm{n}=1,2$, $3,4 \ldots$ where $0<s \leq 1,0<s^{\prime}<1$ and $c$ is the complex plane. Define $z_{1}=(1-s) z+s G_{c}(z)$

$$
z_{n}=(1-s) z_{n-1}+s G_{c}\left(z_{n-1}\right)
$$

Thus, the general escape criterion is $\max \left\{|c|,(2 / s)^{1 / n+1},\left(2 / s^{\prime}\right)^{1 / n+1}\right\}$.

Proof: We shall prove this theorem by induction:

For $\mathrm{n}=1$, we get $G_{c}(z)=z+c$. So, the escape criterion is $|\mathrm{c}|$, which is obvious, i.e. $|z|>\max \{|c|, 0,0\}$

For $\mathrm{n}=2$, we get $G_{c}(z)=z^{2}+c$. So, the escape criterion is $|z|>\max \left\{|c|, 2 / s, 2 / s^{\prime}\right\}$ (See Theorem 3.1)

For n $=3$, we get $G_{c}(z)=z^{3}+c$. So, the result follows from Theorem 3.2 with $\mathrm{a}=0$ and $\mathrm{b}=\mathrm{c}$, such that the escape criterion is $|z|>\max \left\{|c|,(2 / s)^{1 / 2},\left(2 / s^{\prime}\right)^{1 / 2}\right\}$. Hence, the theorem is true for $\mathrm{n}=1,2,3,4 \ldots$

Now, suppose that theorem is true for any $n$. Let $G_{c}(z)=z^{n+1}+c$ and $|z| \geq|c|>(2 / s)^{1 / n+1}$ as well as $|z| \geq|c|>\left(2 / s^{\prime}\right)^{1 / n+1}$ exists. Then,

$$
\begin{aligned}
& \left|G_{n}(z)\right|=\left|\left(1-s^{\prime}\right) z+s^{\prime} G_{c}^{\prime}(z)\right| \text { where } G_{c}^{\prime}(z)=z^{n+1}+c \\
& =\left|z-s^{\prime} z+s^{\prime}\left(z^{n+1}+c\right)\right| \\
& \geq\left|s^{\prime} z^{n+1}-s^{\prime} z+z\right|-s^{\prime}|c| \\
& \geq|z|\left(s^{\prime}\left|z^{n}\right|-s^{\prime}+1\left|-s^{\prime}\right| z \mid \quad(\because|z| \geq|c|)\right. \\
& \geq|z|\left\{\left(s^{\prime}\left|z^{n}\right|+\left|s^{\prime}\right|-|1|\right\}-s^{\prime}|z|\right. \\
& \geq|z|\left(s^{\prime}\left|z^{n}\right|+s^{\prime}-1-s^{\prime}\right) \\
& \geq|z|\left(s^{\prime}\left|z^{n}\right|-1\right)
\end{aligned}
$$

Now, $\left|z_{1}\right|=\left|(1-s) z+s G_{n}(z)\right|$

$$
\begin{aligned}
& =|(1-s) z+s| z\left|\left(s^{\prime}\left|z^{n}\right|-1\right)\right| \\
& =|z-s z+s| z\left|\cdot s^{\prime}\right| z^{n}|-s| z|| \\
& \geq(|z|-s|z|)+\left(s s^{\prime}\left|z^{n+1}\right|-s|z|\right) \\
& \geq\left(s s^{\prime}\left|z^{n+1}\right|+|z|\right) \\
& \geq\left|s s^{\prime} z^{n+1}\right|-|z| \\
& \geq|z|\left(s s^{\prime}\left|z^{n}\right|-1\right)
\end{aligned}
$$


Since $|z|>(2 / s)^{1 / n} ;|z|>\left(2 / s^{\prime}\right)^{1 / n}$ and $|z|>\left(2 / s s^{\prime}\right)^{1 / n}$ So $|z|>\left(2 / s s^{\prime}\right)^{1 / n}$, therefore $\left(s s^{\prime}\left|z^{n}\right|-1\right)>1$

Hence, for some $\lambda>0$, we have $\left(s s^{\prime}\left|z^{n}\right|-1\right)>1+\lambda$.

Thus, $\quad\left|z_{1}\right|>(1+\lambda)|z|$

$$
\begin{aligned}
& \vdots \\
& \left|z_{n}\right|=(1+\lambda)^{n}|z|
\end{aligned}
$$

Therefore, the Ishikawa orbit of $\mathrm{z}$ under the iteration of $z^{n+1}+c$ tends to infinity. Hence $|z|>\max \left\{|c|,(2 / s)^{1 / n},\left(2 / s^{\prime}\right)^{1 / n}\right\}$ is the escape criterion. This proves the theorem.

Corollary 3.7: $\quad$ Suppose that $|c|>(2 / s)^{1 / n-1}$ and $|c|>\left(2 / s^{\prime}\right)^{1 / n-1}$ exists. Then, the Relative Superior orbit $R S O\left(G_{c}, 0, s, s^{\prime}\right)$ escapes to infinity.

\section{Corollary3.8:}

Assume that $\left|z_{k}\right|>\max \left\{|c|,(2 / s)^{1 / k-1},\left(2 / s^{\prime}\right)^{1 / k-1}\right\}$ for some $k \geq 0$. Then $\left|z_{k+1}\right|>\gamma\left|z_{k}\right|$ and $\left|z_{n}\right| \rightarrow \infty$, as $n \rightarrow \infty$. This corollary gives an algorithm for computing the Relative Superior Julia sets for the functions of the form $G_{c}(z)=z^{n}+c, \mathrm{n}$ $=1,2,3,4 \ldots$

\section{FIXED POINTS}

\subsection{Fixed points of quadratic polynomial}

Table 1: Orbit of $F(z)$ for $\left(z_{0}=-\mathbf{1 . 0 7 7 5 6 0 9 7 3 ~ - ~ 0 . 8 2 3 7 6 1 9 1 2 i ~}\right)$ at $s^{\prime}=0.1$ and $s^{\prime}=0.4$

\begin{tabular}{|c|c|c|c|}
\hline $\begin{array}{c}\text { Number of } \\
\text { iteration } \boldsymbol{i}\end{array}$ & $|\mathbf{F}(\mathbf{z})|$ & $\begin{array}{c}\text { Number of } \\
\text { iteration } \boldsymbol{i}\end{array}$ & $|\mathbf{F}(\mathbf{z})|$ \\
\hline 1 & 1.3564 & 16 & 1.716 \\
\hline 2 & 1.0463 & 17 & 1.7164 \\
\hline 3 & 0.94717 & 18 & 1.7166 \\
\hline 4 & 1.0745 & 19 & 1.7167 \\
\hline 5 & 1.3067 & 20 & 1.7167 \\
\hline 6 & 1.5394 & 21 & 1.7167 \\
\hline 7 & 1.7178 & 22 & 1.7167 \\
\hline 8 & 1.8123 & 23 & 1.7167 \\
\hline 9 & 1.8205 & 24 & 1.7167 \\
\hline 10 & 1.7819 & 25 & 1.7167 \\
\hline 11 & 1.7454 & 26 & 1.7167 \\
\hline 12 & 1.7258 & 27 & 1.7167 \\
\hline 13 & 1.718 & 28 & 1.7167 \\
\hline 14 & 1.7158 & 29 & 1.7167 \\
\hline 15 & 1.7156 & 30 & 1.7167 \\
\hline
\end{tabular}

Here we observe that the value converges to a fixed point after 19 iterations
Figure 1. Orbit of $F(z)$ for $\left(\mathbf{z}_{0}=\mathbf{- 1 . 0 7 7 5 6 0 9 7 3 - 0 . 8 2 3 7 6 1 9 1 2 i}\right)$ at $\mathrm{s}^{\prime}=\mathbf{0 . 1}$ and $\mathrm{s}^{\prime}=0.4$

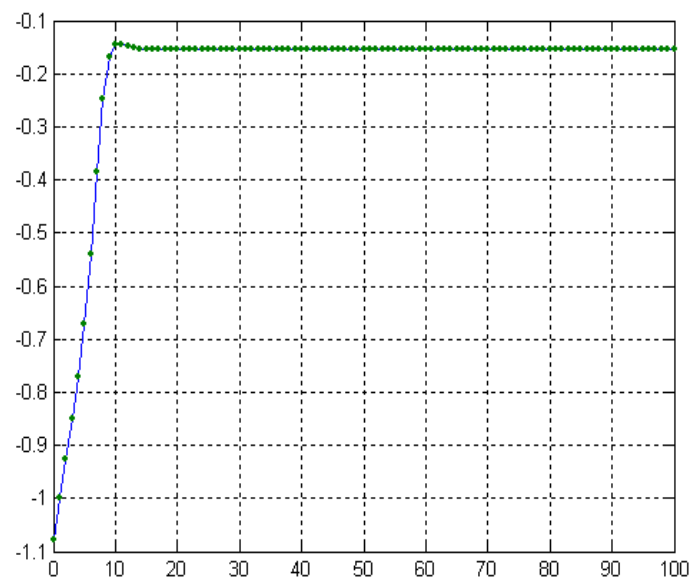

Table 2: Orbit of $F(z)$ for $\left(\mathrm{z}_{0}=\mathbf{- 1 . 7 1} \mathbf{- 0 . 2 4 i}\right)$ at $\mathbf{s}=\mathbf{0 . 3}$ and $\mathrm{s}^{\prime}=\mathbf{0 . 4}$

\begin{tabular}{|c|c|c|c|}
\hline $\begin{array}{l}\text { Number of } \\
\text { iteration i }\end{array}$ & $|\mathrm{F}(\mathrm{z})|$ & $\begin{array}{l}\text { Number of } \\
\text { iteration } \mathrm{i}\end{array}$ & $|\mathrm{F}(\mathrm{z})|$ \\
\hline 1 & 1.7268 & 16 & 0.97304 \\
\hline 2 & 1.3866 & 17 & 0.97304 \\
\hline 3 & 1.2095 & 18 & 0.97304 \\
\hline 4 & 1.132 & 19 & 0.97304 \\
\hline 5 & 1.0602 & 20 & 0.97304 \\
\hline 6 & 1.0051 & 21 & 0.97304 \\
\hline 7 & 0.98257 & 22 & 0.97304 \\
\hline 8 & 0.97564 & 23 & 0.97304 \\
\hline 9 & 0.97371 & 24 & 0.97304 \\
\hline 10 & 0.9732 & 25 & 0.97304 \\
\hline 11 & 0.97308 & 26 & 0.97304 \\
\hline 12 & 0.97305 & 27 & 0.97304 \\
\hline 13 & 0.97304 & 28 & 0.97304 \\
\hline 14 & 0.97304 & 29 & 0.97304 \\
\hline 15 & 0.97304 & 30 & 0.97304 \\
\hline
\end{tabular}

Here we observe that the value converges to a fixed point after 13 iterations

Figure 2. Orbit of $F(z)$ for $\left(z_{0}=-\mathbf{1 . 7 1}-\mathbf{0 . 2 4 i}\right)$ at $\mathbf{s}=\mathbf{0 . 3}$ and $\mathrm{s}^{\prime}=\mathbf{0 . 4}$

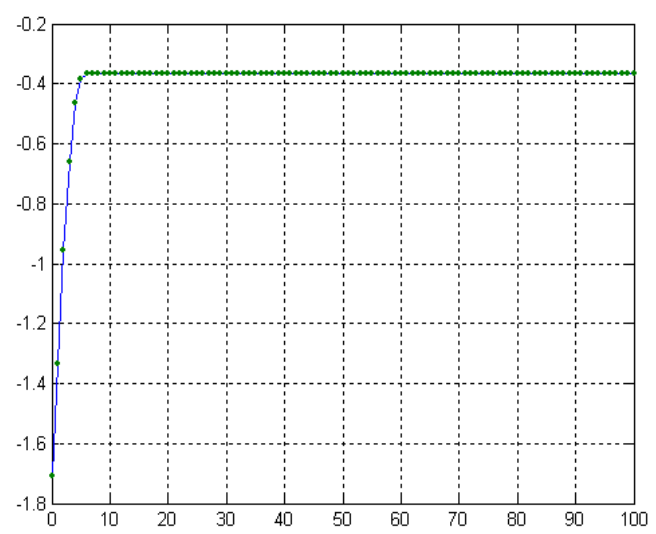


Table 3: Orbit of $F(z)$ for $\left(z_{0}=-4.85-0.86 i\right)$ at $s=0.4$ and $s^{\prime}=0.1$

\begin{tabular}{|c|c|c|c|}
\hline $\begin{array}{c}\text { Number of } \\
\text { iteration } \mathrm{i}\end{array}$ & $|\mathrm{F}(\mathrm{z})|$ & $\begin{array}{c}\text { Number of } \\
\text { iteration } \mathrm{i}\end{array}$ & $|\mathrm{F}(\mathrm{z})|$ \\
\hline 1 & 4.9257 & 16 & 0.69181 \\
\hline 2 & 1.6058 & 17 & 0.69174 \\
\hline 3 & 0.30906 & 18 & 0.69164 \\
\hline 4 & 0.48244 & 19 & 0.69161 \\
\hline 5 & 0.71099 & 20 & 0.69163 \\
\hline 6 & 0.78288 & 21 & 0.69165 \\
\hline 7 & 0.73382 & 22 & 0.69165 \\
\hline 8 & 0.68229 & 23 & 0.69165 \\
\hline 9 & 0.67418 & 24 & 0.69165 \\
\hline 10 & 0.68627 & 25 & 0.69165 \\
\hline 11 & 0.69467 & 26 & 0.69165 \\
\hline 12 & 0.69487 & 27 & 0.69165 \\
\hline 13 & 0.69224 & 28 & 0.69165 \\
\hline 14 & 0.6909 & 29 & 0.69165 \\
\hline 15 & 0.69109 & 30 & 0.69165 \\
\hline
\end{tabular}

Here the value converges to a fixed point after 21 iterations

Figure 3. Orbit of $F(z)$ for $\left(\mathrm{z}_{0}=\mathbf{- 4 . 8 5}-\mathbf{0 . 8 6 i}\right)$ at $\mathbf{s}=\mathbf{0 . 4}$ and $s^{\prime}=\mathbf{0 . 1}$

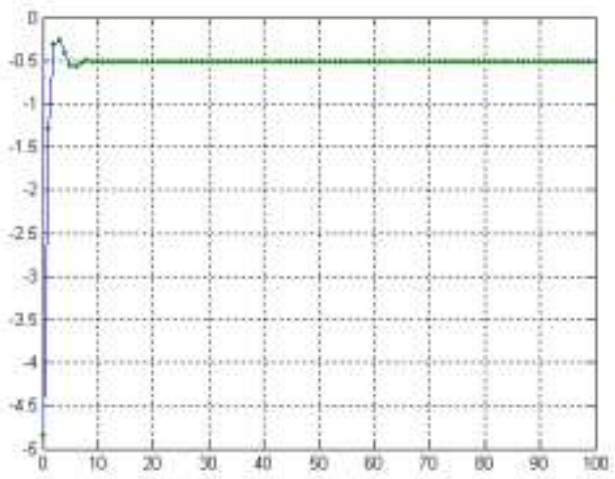

4.2 Fixed points of Cubic polynomial

Table 1: Orbit of $F(z)$ for $\left(z_{0}=\mathbf{- 0 . 0 8 2 + 0 . 0 5 6 i}\right)$ at $s=0.6$ and $\mathrm{s}^{\prime}=\mathbf{0 . 4}$

\begin{tabular}{|c|c|c|c|}
\hline $\begin{array}{c}\text { Number of } \\
\text { iteration } i\end{array}$ & $|\mathrm{~F}(\mathrm{z})|$ & $\begin{array}{c}\text { Number of } \\
\text { iteration } i\end{array}$ & $|\mathrm{~F}(\mathrm{z})|$ \\
\hline 1 & 0.099298 & 16 & 0.4428 \\
\hline 2 & 0.43343 & 17 & 0.4428 \\
\hline 3 & 0.44234 & 18 & 0.4428 \\
\hline 4 & 0.44277 & 19 & 0.4428 \\
\hline 5 & 0.44279 & 20 & 0.4428 \\
\hline 6 & 0.4428 & 21 & 0.4428 \\
\hline 7 & 0.4428 & 22 & 0.4428 \\
\hline 8 & 0.4428 & 23 & 0.4428 \\
\hline 9 & 0.4428 & 24 & 0.4428 \\
\hline 10 & 0.4428 & 25 & 0.4428 \\
\hline 11 & 0.4428 & 26 & 0.4428 \\
\hline 12 & 0.4428 & 27 & 0.4428 \\
\hline 13 & 0.4428 & 28 & 0.4428 \\
\hline 14 & 0.4428 & 29 & 0.4428 \\
\hline 15 & 0.4428 & 30 & 0.4428 \\
\hline
\end{tabular}

Here the value converges to a fixed point after 06 iterations
Figure 1 Orbit of $F(z)$ for $\left(z_{0}=\mathbf{- 0 . 0 8 2 + 0 . 0 5 6 i )}\right.$ at $\mathbf{s}=\mathbf{0 . 6}$ and $\mathrm{s}^{\prime}=\mathbf{0 . 4}$

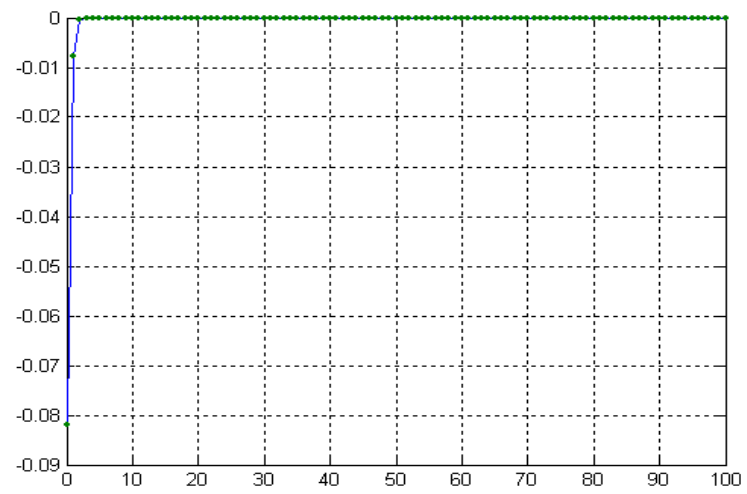

Table 2: Orbit of $\mathrm{F}(\mathrm{z})$ for $\left(\mathrm{z}_{0}=\mathbf{- 0 . 0 8 + 0 . 0 5 7 i )}\right.$ at $\mathrm{s}=\mathbf{0 . 8}$ and $\mathrm{s}^{\prime}=\mathbf{0 . 2}$

\begin{tabular}{|c|c|c|c|}
\hline $\begin{array}{c}\text { Number of } \\
\text { iteration } \mathrm{i}\end{array}$ & $|\mathrm{F}(\mathrm{z})|$ & $\begin{array}{c}\text { Number of } \\
\text { iteration } \mathrm{i}\end{array}$ & $|\mathrm{F}(\mathrm{z})|$ \\
\hline 10 & 0.26107 & 26 & 0.26716 \\
\hline 11 & 0.27154 & 27 & 0.26721 \\
\hline 12 & 0.26406 & 28 & 0.26717 \\
\hline 13 & 0.26942 & 29 & 0.2672 \\
\hline 14 & 0.26558 & 30 & 0.26718 \\
\hline 15 & 0.26833 & 31 & 0.26719 \\
\hline 16 & 0.26637 & 32 & 0.26718 \\
\hline 17 & 0.26777 & 33 & 0.26719 \\
\hline 18 & 0.26677 & 34 & 0.26719 \\
\hline 19 & 0.26749 & 35 & 0.26719 \\
\hline 20 & 0.26697 & 36 & 0.26719 \\
\hline 21 & 0.26734 & 37 & 0.26719 \\
\hline 22 & 0.26708 & 38 & 0.26719 \\
\hline 24 & 0.26727 & 39 & 0.26719 \\
\hline 25 & 0.26713 & 40 & 0.26719 \\
\hline
\end{tabular}

We skipped 09 iterations and after 33 iterations value converges

Figure 2. Orbit of $F(z)$ for $\left(\mathbf{z}_{0}=-\mathbf{0 . 0 8 + 0 . 0 5 7 i )}\right.$ at $\mathbf{s}=\mathbf{0 . 8}$ and $\mathrm{s}^{\prime}=\mathbf{0 . 2}$

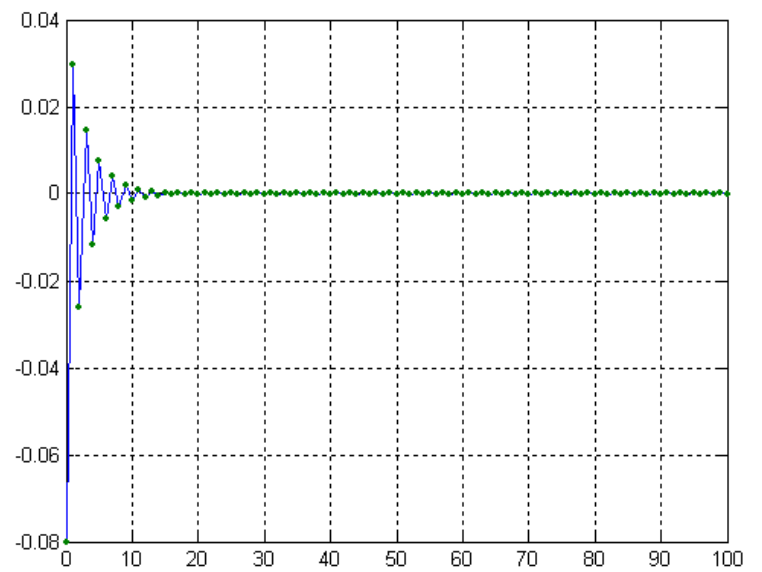


Table 3: Orbit of $F(z)$ for $\left(z_{0}=\mathbf{0 . 1 4 + 2 . 2 5 i}\right)$ at $s=0.4$ and $s^{\prime}=0.1$

\begin{tabular}{|c|c|c|c|}
\hline $\begin{array}{c}\text { Number of } \\
\text { iteration } \mathrm{i}\end{array}$ & $|\mathrm{F}(\mathrm{z})|$ & $\begin{array}{c}\text { Number of } \\
\text { iteration } \mathrm{i}\end{array}$ & $|\mathrm{F}(\mathrm{z})|$ \\
\hline 1 & 2.2544 & 16 & 0.47644 \\
\hline 2 & 0.81868 & 17 & 0.47644 \\
\hline 3 & 0.42429 & 18 & 0.47644 \\
\hline 4 & 0.51067 & 19 & 0.47644 \\
\hline 5 & 0.46793 & 20 & 0.47644 \\
\hline 6 & 0.47848 & 21 & 0.47644 \\
\hline 7 & 0.47594 & 22 & 0.47644 \\
\hline 8 & 0.47655 & 23 & 0.47644 \\
\hline 9 & 0.47641 & 24 & 0.47644 \\
\hline 10 & 0.47644 & 25 & 0.47644 \\
\hline 11 & 0.47643 & 26 & 0.47644 \\
\hline 12 & 0.47644 & 27 & 0.47644 \\
\hline 13 & 0.47644 & 28 & 0.47644 \\
\hline 14 & 0.47644 & 29 & 0.47644 \\
\hline 15 & 0.47644 & 30 & 0.47644 \\
\hline
\end{tabular}

Here the value converges to a fixed point after 12 iterations

Figure 3. Orbit of $F(z)$ for $\left(z_{0}=0.14+2.25 i\right)$ at $s=0.4$ and $s^{\prime}=0.1$

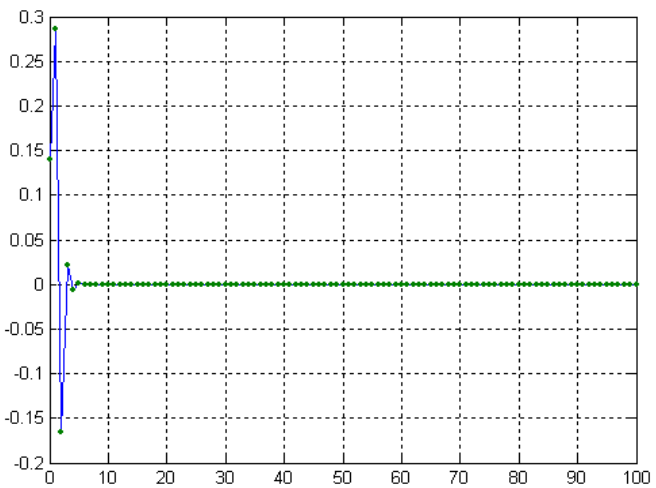

4.3 Fixed points of Bi-quadratic polynomial

Table 1: Orbit of $F(z)$ for $\left(z_{0}=\mathbf{- 0 . 0 4 6 + 0 . 1 6 5 i}\right)$ at $\mathbf{s}=\mathbf{0 . 6}$ and $\mathrm{s}^{\prime}=\mathbf{0 . 4}$

\begin{tabular}{|c|c|c|c|}
\hline $\begin{array}{c}\text { Number of } \\
\text { iteration } i\end{array}$ & $|\mathrm{~F}(\mathrm{z})|$ & $\begin{array}{c}\text { Number of } \\
\text { iteration } i\end{array}$ & $|\mathrm{~F}(\mathrm{z})|$ \\
\hline 1 & 0.17129 & 16 & 0.62734 \\
\hline 2 & 0.58304 & 17 & 0.62734 \\
\hline 3 & 0.69194 & 18 & 0.62734 \\
\hline 4 & 0.64174 & 19 & 0.62734 \\
\hline 5 & 0.63002 & 20 & 0.62734 \\
\hline 6 & 0.62746 & 21 & 0.62734 \\
\hline 7 & 0.6272 & 22 & 0.62734 \\
\hline 8 & 0.62728 & 23 & 0.62734 \\
\hline 9 & 0.62732 & 24 & 0.62734 \\
\hline 10 & 0.62733 & 25 & 0.62734 \\
\hline 11 & 0.62734 & 26 & 0.62734 \\
\hline 12 & 0.62734 & 27 & 0.62734 \\
\hline 13 & 0.62734 & 28 & 0.62734 \\
\hline 14 & 0.62734 & 29 & 0.62734 \\
\hline 15 & 0.62734 & 30 & 0.62734 \\
\hline
\end{tabular}

Here the value converges to a fixed point after 11 iterations
Figure 1 Orbit of $\mathrm{F}(\mathrm{z})$ for $\left(\mathrm{z}_{0}=\mathbf{- 0 . 0 4 6 + 0 . 1 6 5 i}\right)$ at $\mathrm{s}=\mathbf{0 . 6}$ and $\mathrm{s}^{\prime}=\mathbf{0 . 4}$

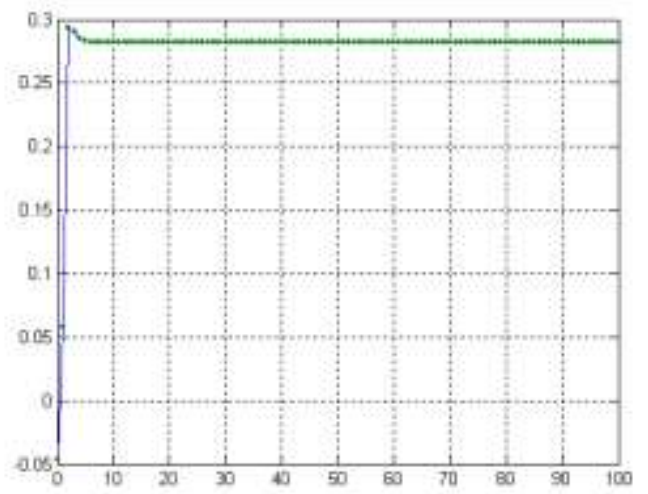

Table 2: Orbit of $\mathrm{F}(\mathrm{z})$ for $\left(\mathrm{z}_{0}=\mathbf{0 . 1 3 4 + 0 . 1 2 8 i}\right)$ at $\mathrm{s}=\mathbf{0 . 3}$ and $\mathrm{s}^{\prime}=\mathbf{0 . 4}$

\begin{tabular}{|c|c|c|c|}
\hline $\begin{array}{c}\text { Number of } \\
\text { iteration } \mathrm{i}\end{array}$ & $|\mathrm{F}(\mathrm{z})|$ & $\begin{array}{c}\text { Number of } \\
\text { iteration } \mathrm{i}\end{array}$ & $|\mathrm{F}(\mathrm{z})|$ \\
\hline 81 & 0.9200 & 96 & 0.9199 \\
\hline 82 & 0.9196 & 97 & 0.9200 \\
\hline 83 & 0.9197 & 98 & 0.9200 \\
\hline 84 & 0.9201 & 99 & 0.9198 \\
\hline 85 & 0.9201 & 100 & 0.9198 \\
\hline 86 & 0.9198 & 101 & 0.9200 \\
\hline 87 & 0.9197 & 102 & 0.9200 \\
\hline 88 & 0.9200 & 103 & 0.9199 \\
\hline 89 & 0.9201 & 104 & 0.9198 \\
\hline 90 & 0.9199 & 105 & 0.9199 \\
\hline 91 & 0.9197 & 106 & 0.9200 \\
\hline 92 & 0.9199 & 107 & 0.9199 \\
\hline 93 & 0.9200 & 108 & 0.9199 \\
\hline 94 & 0.9199 & 109 & 0.9199 \\
\hline 95 & 0.9198 & 110 & 0.9199 \\
\hline
\end{tabular}

We skipped 81 iterations and after 107 iterations value converges

Figure 2 Orbit of $\mathrm{F}(\mathrm{z})$ for $\left(\mathrm{z}_{0}=\mathbf{0 . 1 3 4 + 0 . 1 2 8 i}\right)$ at $\mathbf{s}=\mathbf{0 . 3}$ and $\mathrm{s}^{\prime}=\mathbf{0 . 4}$

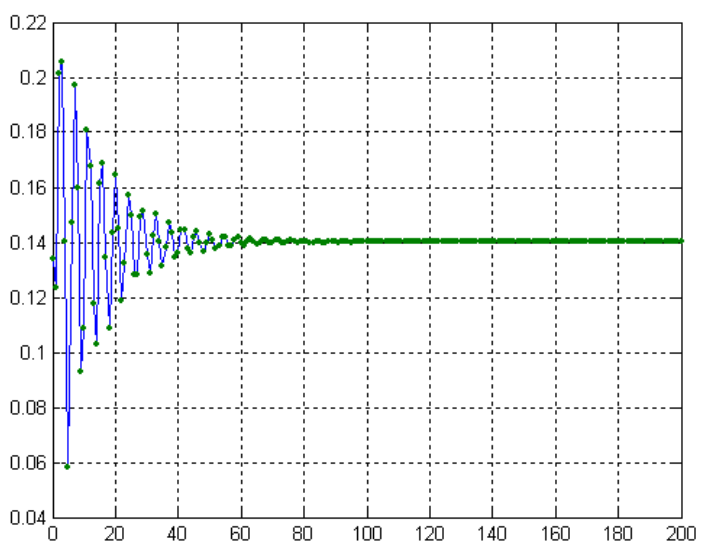


Table 3: Orbit of $F(z)$ for $\left(z_{0}=-\mathbf{0 . 1 1 8 + 0 . 0 2 1 i}\right)$ at $s=0.4$ and $\mathrm{s}^{\prime}=\mathbf{0 . 1}$

\begin{tabular}{|c|c|c|c|}
\hline $\begin{array}{c}\text { Number of } \\
\text { iteration i }\end{array}$ & $|\mathrm{F}(\mathrm{z})|$ & $\begin{array}{c}\text { Number of } \\
\text { iteration } \mathrm{i}\end{array}$ & $|\mathrm{F}(\mathrm{z})|$ \\
\hline 30 & 0.56705 & 45 & 0.56824 \\
\hline 31 & 0.56825 & 46 & 0.56826 \\
\hline 32 & 0.56908 & 47 & 0.5683 \\
\hline 33 & 0.56782 & 48 & 0.56827 \\
\hline 34 & 0.56801 & 49 & 0.56826 \\
\hline 35 & 0.56872 & 50 & 0.56828 \\
\hline 36 & 0.56819 & 51 & 0.56827 \\
\hline 37 & 0.56803 & 52 & 0.56826 \\
\hline 38 & 0.56847 & 53 & 0.56828 \\
\hline 39 & 0.56831 & 54 & 0.56828 \\
\hline 40 & 0.56812 & 55 & 0.56827 \\
\hline 41 & 0.56833 & 56 & 0.56827 \\
\hline 42 & 0.56834 & 57 & 0.56827 \\
\hline 43 & 0.5682 & 58 & 0.56827 \\
\hline 44 & 0.56827 & 59 & 0.56827 \\
\hline
\end{tabular}

We skipped 29 iterations and after 55 iterations value converges

Figure 3. Orbit of $F(z)$ for $\left(z_{0}=\mathbf{- 0 . 1 1 8 + 0 . 0 2 1 i}\right)$ at $\mathbf{s}=\mathbf{0 . 4}$ and $s^{\prime}=0.1$

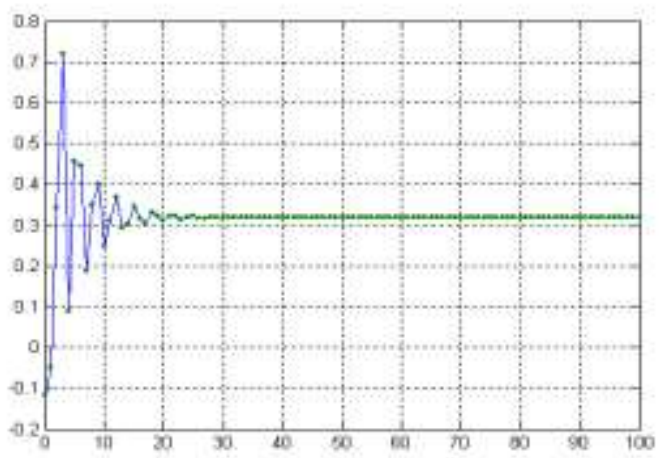

5. GENERATION OF RELATIVE SUPERIOR JULIA SETS:

We generated the Relative Superior Julia sets. We present here some beautiful filled Relative Superior Julia sets for quadratic, cubic and biquadratic function.

5.1 Relative Superior Julia sets for Quadratic:

Figure 1: Relative Superior Julia Set for $s^{\prime}=s^{\prime}=1, c=\mathbf{- 1 . 3 8}$

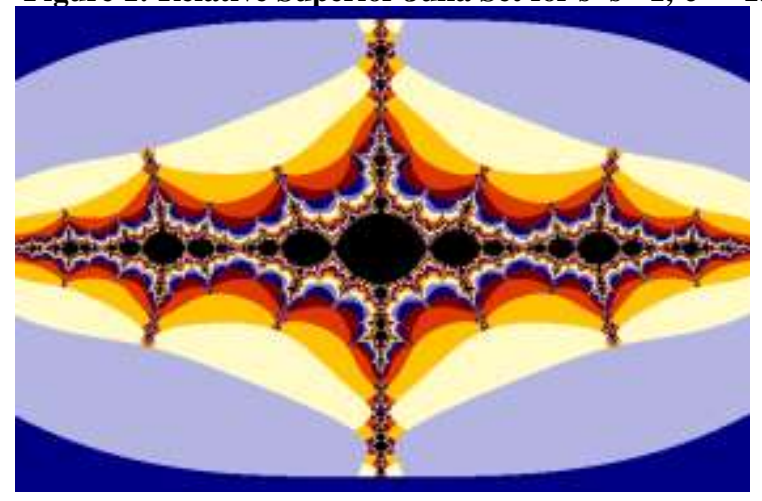

Figure 2: Relative Superior Julia Set for $s^{\prime}=1, s^{\prime}=0.3$,

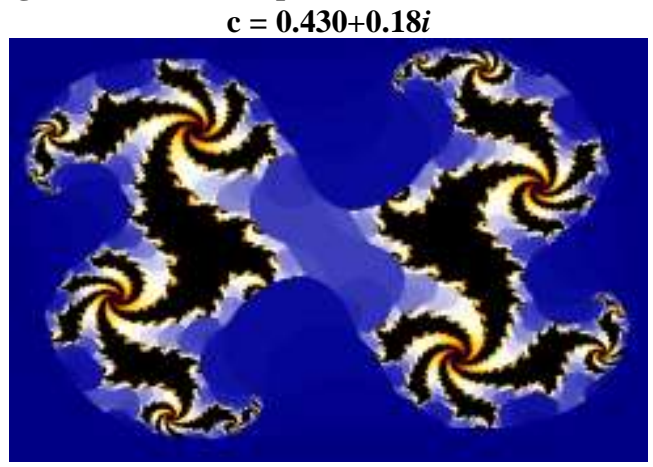

Figure 3: Relative Superior Julia Set for $s=0.3, s^{\prime}=1$,

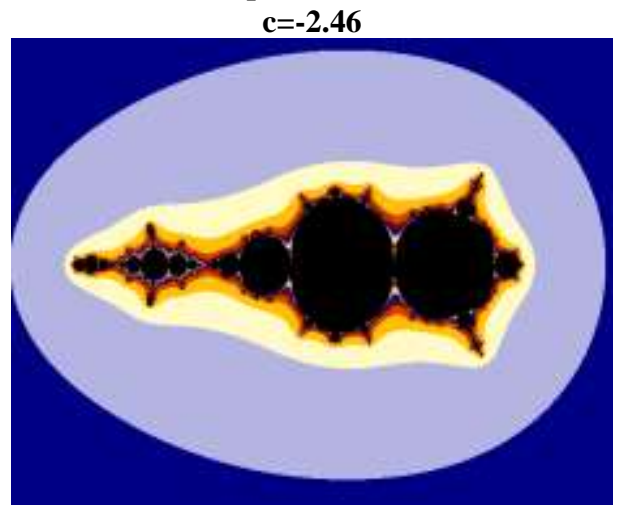

Figure 4: Relative Superior Julia Set for $s=0.1, s^{\prime}=0.4$,

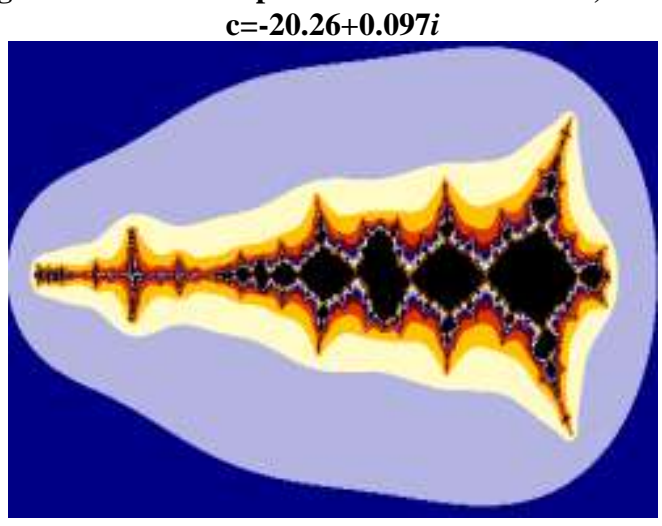

Figure 5: Relative Superior Julia Set for $s=0.4, s^{\prime}=0.1$,

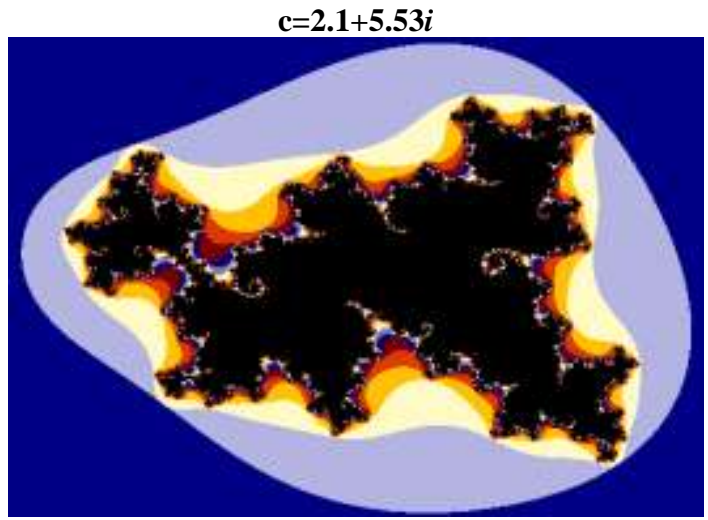


5.2 Relative Superior Julia sets for Cubic function:

Figure 1: Relative Superior Julia for $s^{\prime}=s^{\prime}=1, c=-0.2+1.1 i$

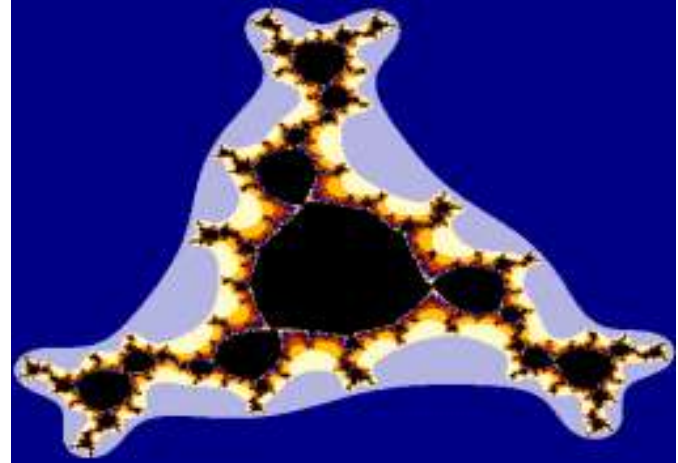

Figure 2: Relative Superior Julia Set for $s=1, s^{\prime}=0.5$,

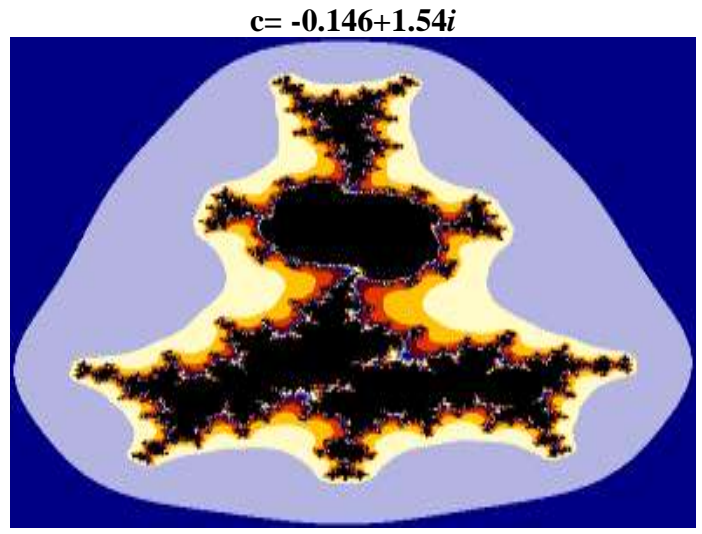

Figure 3: Relative Superior Julia Set for $s=0.3, s^{\prime}=1, c=$ -

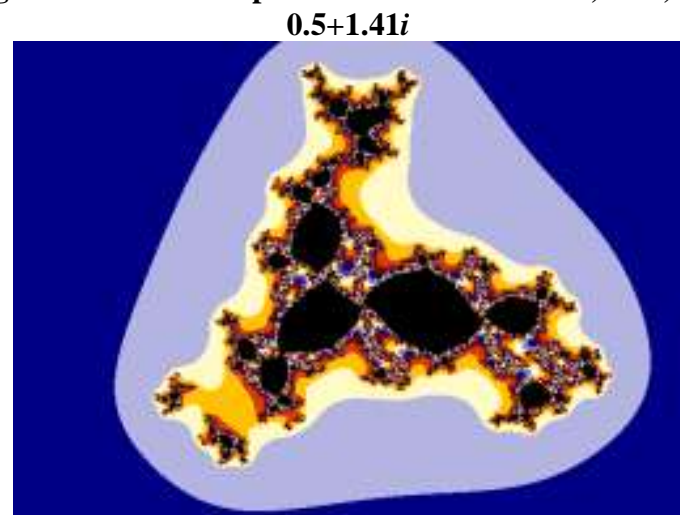

Figure 4: Relative Superior Julia Set for $s^{=0.1}, s^{\prime}=0.4$,

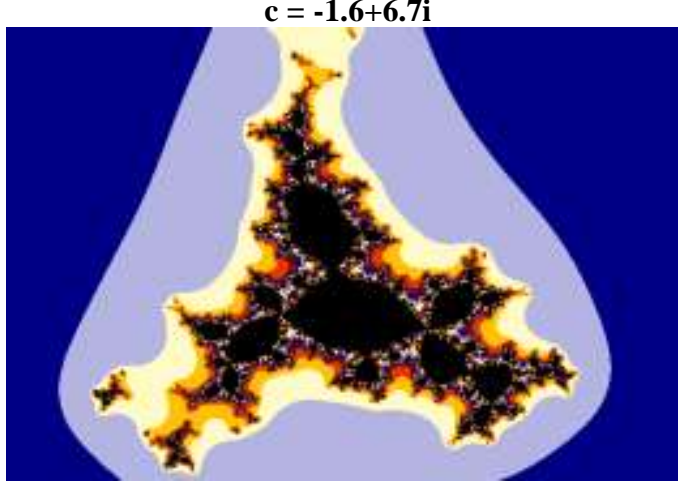

Figure 5: Relative Superior Julia Set for $s=0.4, s^{\prime}=0.1$,

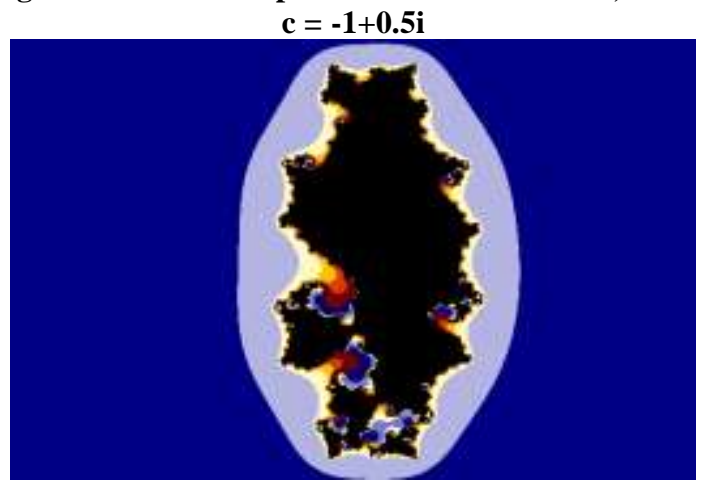

5.3 Relative Superior Julia sets for Bi-quadratic function:

Figure 1: Relative Superior Julia for $s^{\prime}=s^{\prime}=1, c=0.58-0.98 i$

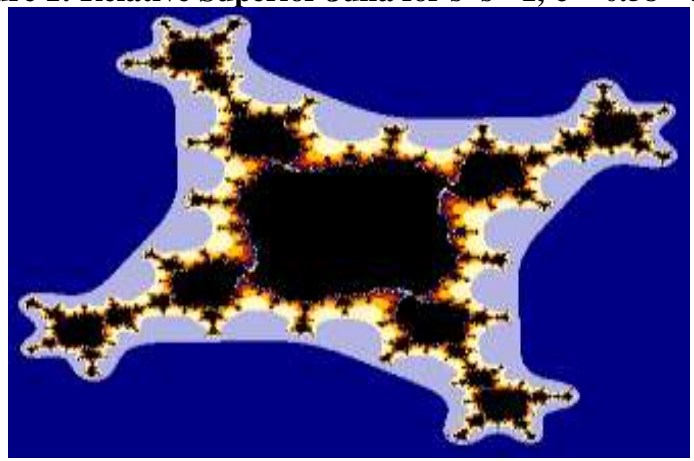

Figure 2: Relative Superior Julia Set for $s=1, s^{\prime}=0.5, c=-1.57$

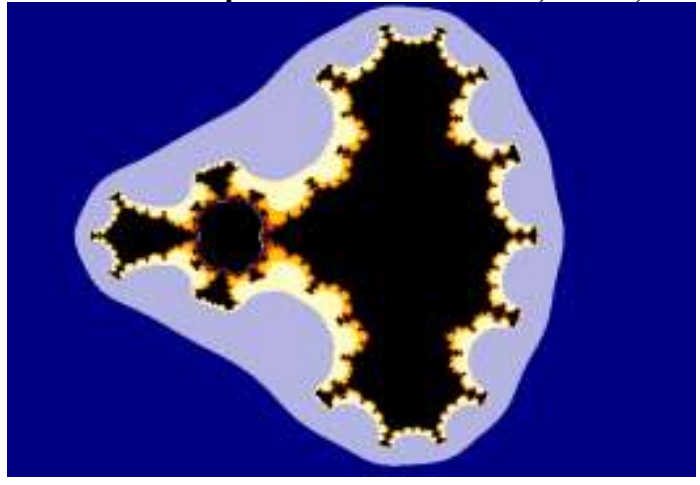

Figure 3: Relative Superior Julia Set for $s=0.5, s^{\prime}=1, c=-1.24$

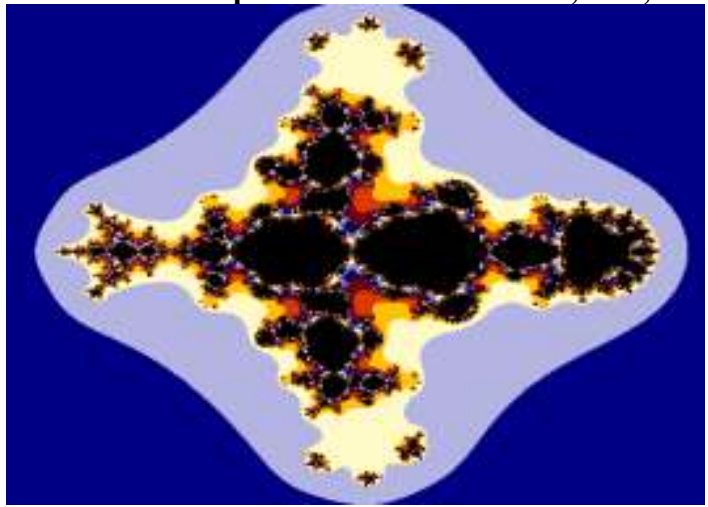


Figure 4: Relative Superior Julia Set for $s=0.1, s^{\prime}=0.4$,

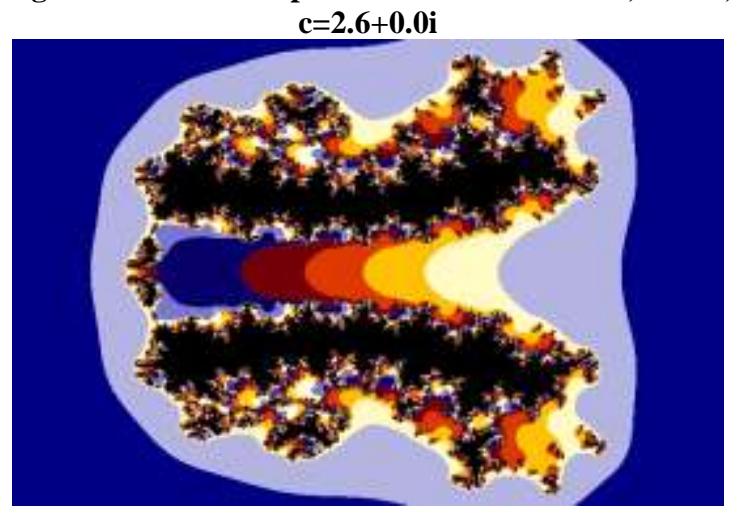

Figure 5: Relative Superior Julia Set for $s=0.3, s^{\prime}=0.4, c=$ -

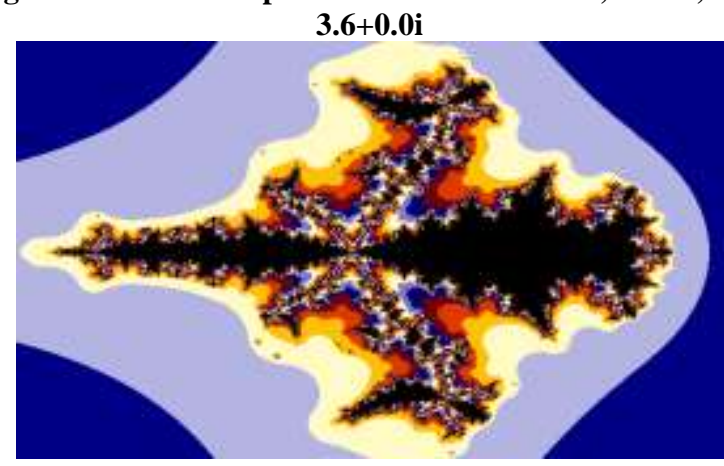

\section{REFERENCES}

[1] R.Abraham and C.Shaw, Dynamics: "The Geometry of Behaviour, Part One: Periodic Behavior, Part Two: Chaotic Behavior", Aerial Press, Santa Cruz, Calif, (1982).

[2] Alan F.Beardon, "Iteration of Rational functions", Springer Verlag, N.York, Inc.(1991).

[3] V.Berinde, "Iterative approximation of fixed points", Editura Efermeide, Baia Mare, (2002).

[4] S,Beddings and K.Briggs, "Itreation of quaterinian maps", Int J. Bifur Chaos. Appl. Sci. and Engg.5 (1995), 877-881.

[5] B.Branner and J.Hubbard, "The Iteration of Cubic Polynomials". Part I,Acta. Math. 66(1998), 143-206.

[6] G.V.R.Babu and K.N.V.Prasad, "Mann iteration converges faster than Ishikawa iteration for the class of Zamfirescu operators", Fixed Point Theory and its Applications, Vol. 2006, Art. ID 49615, 1-6(2006).

[7] P.W.Carlson, "Pseudo $3 D$ rendering methods for fractals in the complex plane, Computer and Graphics", 20(5), (1996), 757-758.
[8] R.M.Crownover, "Introduction to Fractals and Chaos, Mandelbrot sets", Jones and Barlett Publishers, (1995).

[9] R.L.Daveney, “An Introduction to Chaotic Dynamical Systems", Springer-Verlag, N.York.Inc.1994.

[10] K.Heinz, Becker and Michael Dorfler, "Dynamical Systems and Fractals", Cambridge Univ. Press, 1989.

[11] R.A.Hohngren, "A First Course in Discrete Dynamical Systems", Springer-Verlag, 1994.

[12] S.Ishikawa, "Fixed points by a new iteration method", Proc. Amer. Math. Soc.44 (1974), 147-150.

[13] M.A.Kransnosel, "Two remarks on method of successive approximations", Uspehi. Math. Nauk. 10(1995), 123-127.

[14] W.R.Mann, "Mean Value methods in iteration", Proc. Amer. Math. Soc.4 (1953), 506-510.

[15] B.B.Mandelbrot, "The Fractal aspects of iteration of $z \rightarrow \lambda z(1-z)$ for complex $\lambda$ and $z$," Ann. N. Y. Acad. Sci. 357(1980), 249-259.

[16] J.R.Munkers, “Topology: A First course “, Prantice Hall of India Publ. Ltd., N.Delhi, 1988.

[17] M.O.Osilike, "Stability results for Ishikawa fixed point iteration procedure", Indian Journal of Pure and Appl. Math., 26(1995), 937-945.

[18] M.O.Osilike, "Iterative construction of fixed points of multivalued operators of the accretive type", Sochow J.Math. 22(1996), 85-92.

[19] H.Peitgen, H.Jurgens and D.Saupe, "Fractals for classroom Part Two, Complex Systems AND Mandelbrot Sets", Springer-Verlag, N.York, Inc. 1992.

[20] H.Peitgen, H.Jurgens and D.Saupe," Chaos and Fractals", Springer-Verlag, N.York, Inc. 1992.

[21] M.Rani and V.Kumar, "Superior Mandelbrot Set", J Korea Soc. Math. Edu. Series, DResearch in Maths. Edu. no.4,8(2004), 279-291.

[22] B.E.Rhoades, "Some fixed point iterations procedures", Int.J.Math.Sci. 14(1991), 1-16.

[23] B.E.Rhoades, "Fixed points iterations for certain nonlinear mappings", J .Math. Anal. Appl., 183(1994), 118-120.

[24] B.E.Rhoades and S.M.Solutz, "On the equivalence of Mann and Ishikawa iteration methods", Int J. Math. Sci. no.7 (2003), 451-459.

[25] D.Rochon, "A Generalized Mandelbrot Set for BiComplex Numbers", World Scientific Publishing Company, Fractals 8(4), (2000), 355-368. 